https://doi.org/10.15407/ujpe64.11.1054

V.O. PELYKH, Y.V. TAISTRA

Ya. Pidstryhach Institute for Applied Problems of Mechanics and Mathematics,

Nat. Acad. of Sci. of Ukraine

(3b, Naukova Str., Lviv 79060,Ukraine; e-mail: pelykh@iapmm.lviv.ua,ythelloworld@gmail.com)

\title{
WAVE OPTICS IN THE KERR SPACE-TIME TAKING THE SPIN-HELICITY INTERACTION INTO ACCOUNT ${ }^{1}$
}

\begin{abstract}
We apply an algebraically special solution of the Maxwell equations in the Kerr space-time, which we specify as outgoing in the Chandrasekhar meaning, to obtain the wave vectors of right- and left-polarized waves and prove that the nullity condition of field invariants yield the non-nullity of wave vectors and that the wave vector is not geodesic. We also show how these are related to the analysis of radiation in the Kerr space-time, provided by Starobinskii and Teukolsky.
\end{abstract}

Ke ywords: one-way null field, Maxwell spinor, the Kerr space-time, separation of variables, wave vector, geodesics.

\section{Introduction}

The discovery of black holes by observing gravitational radiation from their merger [1], coalescences of a black hole $(\mathrm{BH})$ and a neutron star [2], and observations of the shadow of supermassive black holes residing at the center of the elliptical galaxy Messier 87 by the Event Horizon Telescope collaboration [3] not only confirm the predictions of the theory of general relativity, but also prove the efficiency of methods developed to analyze such processes, including analytical and numerical ones. However, the possibility of the complete exclusion of the non-Einstein nature of gravity or imitation of Kerr black hole (KBH) by a Schwarzschild $\mathrm{BH}$ or by horizonless compact objects from these observations is not complete at this time [4-6]. Therefore, the need for a further development of analytic methods of study of the field behavior in a vicinity of $\mathrm{KBH}$ as sources of information about the later does not diminish, which makes it possible to describe phenomena in a wide range of changes in coordinates and parameters, and to circumvent the problems of accuracy and convergence of approximate methods, especially in the strong fields.

The basis for such an analytic description of the behavior of electromagnetic fields is the Maxwell equations, whose use in the Riemannian spaces of general

(c) V.O. PELYKH, Y.V. TAISTRA, 2019

1054 relativity is of fundamental complexity. The main one is the coupling of equations - each equation contains derivatives of all unknown functions. This difficulty is absent in the case of a scalar field, which allowed ones ([7] and [8]) to prove the existence of superradiance in the Kerr space-time (KST). Teukolsky [9] partially decoupled the equations for gravitational, electromagnetic, and neutrino fields in the Petrov-type $D$ spacetimes and confirmed the existence of superradiance in the KST also in case of an electromagnetic field. In works [7], [10], and [9], the solutions were obtained with the use of the expansion in series in spin-weighted spheroidal harmonics. Those and all other approaches described in detail in [11] gave approximate solutions in the form of series in a different system of functions with coefficients determined by three-term recursion relations. Although the solutions can be obtained now with arbitrary accuracy under the application of numerical methods, their use in all ranges of source characteristics (mass and angular momentum), variables, and separation constants remains complicated. Alternatively, the approximate analytic solutions can be obtained at two ends of the frequency range - in the high- or low-frequency range (see, e.g., [12] and because of complexity references therein) and often contain errors.

1 This work is based on the results presented at the XI BolyaiGauss-Lobachevskii (BGL-2019) Conference: Non-Euclidean, Noncommutative Geometry and Quantum Physics.

ISSN 2071-0194. Ukr. J. Phys. 2019. Vol. 64, No. 11 
Modeling the gravitational field with a material medium and confining himself to qualitative remarks, Mashhoon [13] noted for the first time that incident right circularly polarized photons must scatter differently from left-circularly polarized ones due to the interaction of the photon spin with the gravitational field (Mashhoon effect).

In the geometric optics approximation, Guadagnini [18] and Barbieri and Guadagnini [19] have computed the helicity asymmetry which is associated with the polarization of light at the gravitational deflection of light by a rotating body. The polarization dependence of photon trajectories by using the modified geometric optics approximation was calculated by Frolov and Shoom [20]. Mashhoon's prediction of the interaction of the photon spin with the gravitational field was confirmed by Asenjo and Hojman [21] exploiting an exact solution in the case of electromagnetic waves on the Gödel space-time and an approximate solution on the Kerr one.

We will aim to obtain exact results regarding the behavior in the Kerr space-time of the wave vector of the null one-way (NOW) Maxwell field, which is algebraically special and outgoing, according to the Chandrasekhar terminology. In doing so, we will use a corresponding analytic solution to the Maxwell equations [14-16]. Two remarks are needed regarding this solution. First, of course, it is partial, but this does not prevent it from describing the most common patterns of propagation of electromagnetic radiation, that is inherent in any other solution. Second, the solution is singular on the rotation axis $\theta=0, \theta=\pi$ (even in a flat space-time). However, it is known that the singularity on the rotation axis is not invariant, and this solution in the Boyer-Lindquist coordinates as a null one-way is physically meaningful on the interval $0<\theta<\pi$.

The metric signature is $(+,-,-,-)$. The equations are considered in geometrized units, where $c=G=$ $=1$, and the sufficient smoothness of all functions is supposed, which does not limit physical generality.

\section{Wave Vector of NOW}

\section{Maxwell Field on the Kerr Space-Time}

In [16] in the Kerr space-time, we have considered an electromagnetic field which is a partial case of the algebraically special field. Its principal spinors are multiples and, in addition, are aligned with one of the Weyl repeated principal spinors. If the Weyl repeated principal spinor is aligned to $o_{A}$ spinor, the Maxwell spinor looks like $\varphi_{A B}=\varphi_{2} o_{A} o_{B}$, and the Maxwell field is a NOW field, outgoing by Chandrasekhar [22].

In the Kinnersly gauge, a solution of the Maxwell equations in the form of a NOW field with partially separated variables, which is finite in time and $2 \pi$ periodic in the azimuthal argument, has the form $[15,16]$

$\varphi_{2}=C \frac{e^{i \omega \eta_{1}+i m \eta_{2}+a \omega \cos \theta+m \ln \left(\frac{1-\cos \theta}{\sin \theta}\right)}}{\sin \theta(r-i a \cos \theta)}$,

where

$\eta_{1}=t-r-M \ln \Delta-\frac{M^{2}}{\sqrt{M^{2}-a^{2}}} \ln \left(\frac{r-r_{+}}{r-r_{-}}\right)$,

$\eta_{2}=\phi-\frac{a}{2 \sqrt{M^{2}-a^{2}}} \ln \left(\frac{r-r_{+}}{r-r_{-}}\right)$,

$t, r, \theta, \phi-$ the Boyer-Lindquist coordinates, $M-\mathrm{a}$ Kerr mass parameter, $a-$ an angular momentum per unit mass, $C=C(\omega) \in \mathbb{C}-$ an arbitrary constant, $\omega \in \mathbb{R}, m \in \mathbb{Z}$ - wave frequency and azimuthal number, $\Delta=r^{2}-2 M r+a^{2}, r_{+}=M+\sqrt{M^{2}-a^{2}}$, and $r_{-}=M-\sqrt{M^{2}-a^{2}}$.

The phase of wave (1)

$\Phi=\omega\left(t-r-M \ln \Delta-\frac{M^{2}}{\sqrt{M^{2}-a^{2}}} \ln \left(\frac{r-r_{+}}{r-r_{-}}\right)\right)+$

$+m\left(\phi-\frac{a}{2 \sqrt{M^{2}-a^{2}}} \ln \left(\frac{r-r_{+}}{r-r_{-}}\right)\right)$

defines the wave four-vector with components

$k_{\mu}=\left[\omega,-\frac{\omega\left(r^{2}+a^{2}\right)+a m}{\Delta}, 0, m\right]$

and

$k^{\mu}=k_{\nu} g^{\mu \nu}=\left[\omega\left(1+\frac{2 M r\left(r^{2}+a^{2}+\frac{m a}{\omega}\right)}{\Sigma \Delta}\right)\right.$,

$\frac{\omega\left(r^{2}+a^{2}\right)+a m}{\Sigma}, 0$,

$\left.-\frac{m}{\Delta \sin ^{2} \theta}+\frac{2 M r\left(a \omega \sin ^{2} \theta+m\right)}{\Sigma \Delta \sin ^{2} \theta}\right]$,

and constitutes the dispersion law. As opposed to [21], we know the dynamics of the amplitude and can obtain the scalar square of this vector explicitly:

$k^{\mu} k_{\mu}=-\frac{\left(a \omega \sin ^{2} \theta+m\right)^{2}}{\Sigma \sin ^{2} \theta}$.

It follows that the wave vector is not null generally, and it becomes null only at the spatial infinity, or 
when condition $a \omega \sin ^{2} \theta+m=0$ holds, or in the Schwarzschild space-time for $m=0$. It is not singular on the outer horizon and, unlike the conclusion obtained in [21], cannot be space-like.

The congruence to which the vector field $k_{\mu}$ is tangent is not geodesic:

$$
\begin{aligned}
& k^{\mu} \nabla_{\mu} k_{0}=k^{\mu} \nabla_{\mu} k_{1}=0, \\
& k^{\mu} \nabla_{\mu} k_{2}=r \frac{\left(a \omega \sin ^{2} \theta+m\right)^{2}}{\Sigma^{2} \sin ^{2} \theta}, \\
& k^{\mu} \nabla_{\mu} k_{3}=\frac{\cos \theta}{\Sigma^{2} \sin ^{3} \theta} \times \\
& \times\left(\left(m^{2}-a^{2} \omega^{2} \sin ^{4} \theta\right)\left(r^{2}+a^{2}\right)-\right. \\
& \left.-2 a^{2} m \sin ^{2} \theta\left(a \omega \sin ^{2} \theta+m\right)\right) .
\end{aligned}
$$

As $a \rightarrow 0$,

$k^{\mu} \nabla_{\mu} k_{0}=k^{\mu} \nabla_{\mu} k_{1}=0$,

$k^{\mu} \nabla_{\mu} k_{2}=\frac{m^{2}}{r^{3} \sin ^{2} \theta}$,

$k^{\mu} \nabla_{\mu} k_{3}=\frac{m^{2} \cos \theta}{r^{2} \sin ^{3} \theta}$.

To compare our results with those of Starobinskii and Teukolsky, let us go to the new radial variable "tortoise" coordinate $r^{*}$, which eliminates singularities on the horizon

$\frac{d r^{*}}{d r}=\frac{r^{2}+a^{2}}{\Delta}$

Then the Maxwell tensor and the energy-momentum tensor corresponding to the NOW-solution take the form

$$
\begin{aligned}
& F_{a b}=\sqrt{2} \times \\
& \times\left(\begin{array}{cccc}
0 & -\frac{a P}{r^{2}+a^{2}} & -\frac{1}{\sin \theta} Q & P \\
\frac{a P}{r^{2}+a^{2}} & 0 & \frac{\Sigma Q}{\left(r^{2}+a^{2}\right) \sin \theta} & -P \\
\frac{1}{\sin \theta} Q & -\frac{\Sigma Q}{\left(r^{2}+a^{2}\right) \sin \theta} & 0 & -a \sin \theta Q \\
-P & P & a \sin \theta Q & 0
\end{array}\right), \\
& T_{a b}=\frac{\left|\varphi_{2}\right|^{2}}{2 \pi}\left(\begin{array}{cccc}
1 & -\frac{\Sigma}{r^{2}+a^{2}} & 0 & -a \sin ^{2} \theta \\
-\frac{\Sigma}{r^{2}+a^{2}} & \frac{\Sigma^{2}}{\left(r^{2}+a^{2}\right)^{2}} & 0 & \frac{a \sin ^{2} \theta \Sigma}{r^{2}+a^{2}} \\
0 & 0 & 0 & 0 \\
-a \sin ^{2} \theta & \frac{a \sin ^{2} \theta \Sigma}{r^{2}+a^{2}} & 0 & a^{2} \sin ^{4} \theta
\end{array}\right),
\end{aligned}
$$

where

$P=\left(c_{1} \sin \left(\omega \eta_{1}+m \eta_{2}\right)+c_{2} \cos \left(\omega \eta_{1}+m \eta_{2}\right)\right) \times$

$\times e^{-a \omega \cos \theta}\left(\frac{1-\cos \theta}{\sin \theta}\right)^{m}$,

$Q=\left(c_{1} \cos \left(\omega \eta_{1}+m \eta_{2}\right)-c_{2} \sin \left(\omega \eta_{1}+m \eta_{2}\right)\right) \times$

$\times e^{-a \omega \cos \theta}\left(\frac{1-\cos \theta}{\sin \theta}\right)^{m}$,

and they become regular on the horizon $r_{+}$.

The $k_{r^{\star}}$ component of the wave vector (5) becomes

$k_{r^{*}}=\frac{d \Phi}{d r^{*}}=-\omega-\frac{d}{d r^{*}}\left(\frac{m a}{2 \sqrt{M^{2}-a^{2}}} \ln \frac{r-r_{+}}{r-r_{-}}\right)=$

$=-\omega-\frac{m a}{r^{2}+a^{2}}$,

and we obtain the radial components of the group and phase velocities are as follows: ${ }^{2}$

$v_{\text {gr. }}=-\frac{d k}{d \omega}=-1$

$v_{\text {ph. }}=-\frac{k}{\omega}=-1+\frac{a m}{\omega\left(r^{2}+a^{2}\right)}=-1+m \frac{\Omega(r)}{\omega}$.

Formula (21) exactly defines the critical points (surfaces) for waves outgoing from $\mathrm{KBH}$ - the points, where the phase velocity $v_{\text {ph. }}$ changes its sign (see also $[15,17])$

$r_{\mathrm{cr} .}=\sqrt{\frac{a m}{\omega}-a^{2}}$,

as compared with works [7] and [9] [formula (5.11)], where the results obtained only in a vicinity of the horizon $\left(r \rightarrow r_{+}\right)$

$r_{\mathrm{cr} .}=r_{+}=\sqrt{\frac{a m}{\omega}-a^{2}}$.

Obviously,

$\Omega\left(r_{+}\right)=\frac{a}{r_{+}^{2}+a^{2}}$

is the angular velocity of $\mathrm{KBH}$, which is defined as angular velocity of the horizon.

We conclude from whence that the condition for a change in the wave ingoing-outgoing character at arbitrary point is of the same form as on the horizon.

${ }^{2}$ Hereafter, we change the sign of $\omega$ to compare with the Starobinskii or Teukolsky results.

ISSN 2071-0194. Ukr. J. Phys. 2019. Vol. 64, No. 11 
The group velocity of an outgoing NOW wave is always the speed of light (in vacuum), but the phase velocity is less than the speed of light for frequencies, for which the condition

$\omega<\frac{a m}{\left(r^{2}+a^{2}\right)}$

is fulfilled, and has the opposite direction to the group velocity.

The phase velocity tends to the speed of light, when $\omega \rightarrow \infty$ or $r \rightarrow \infty$, and becomes aligned in the same way as the group velocity. As can be seen from formula (21) which is correct throughout the full frequency range, the phase velocity depends on the sign of $\omega$, which defines the waves with different polarizations. It is the wave-approach manifestation of a spin-helicity interaction.

\section{Conclusions}

It is known that the concepts of group and phase velocities of propagating waves is fully meaningful only in the cases where the medium or space allows the existence of plane waves. This requirement does not meet the Riemannian space-time of general relativity. The use of a spinor representation of the Maxwell equations and the use of the algebraically special solution previously obtained by us in the KST implies that, in particular, in this space-time,

1. the condition that Maxwell field is null,

$F_{a b} F^{a b}=0, \quad \varepsilon_{a b c d} F^{a b} F^{c d}=0$,

does not imply that the wave vector is null;

2 . the influence of the gravitational field of a rotating mass on the Maxwell field determines the frequency dispersion of the wave vector (5);

3 . the group and phase velocities of the electromagnetic wave can be directed in the opposite directions, as it determines relations (20)-(21) between the characteristics of gravitational and electromagnetic fields.

Although the Mariot-Robinson theorem guarantees that if $F_{a b}$ is a null electromagnetic field, then the repeated principal null direction generates a geodesic shearfree null congruence, but, on the other hand, it is known that the motion of particles with spin in a gravitational field is described by the MathissonPapapetrou equation, the solutions of which are not geodesics. Moreover, in the ultrarelativistic case of particle motion, the deviation of the trajectories from the geodesics becomes very significant $[23,24]$. Therefore, the geodesics, whose existence is predicted by the Mariot-Robinson theorem, cannot be associated with wave fronts of electromagnetic waves, and the description of the motion of an electromagnetic wave in terms of wave vectors is more consistent to the physical prediction, even if the wave phase is not a bilinear function of the components of the wave vector and coordinates, as is the case in our solution. The direction and absolute value of the group and phase velocities is determined by relations (20)-(21), which generalize the Starobinskii and Teukolsky ones, are exact, and are valid throughout the space, not just near the outer horizon.

This work was supported by the budget program of Ukraine "Support for the development of priority research areas" (CPCEC 6451230) and by the Scientific Cosmic Program of National Academy of Sciences of Ukraine.

1. B.P. Abbott et al. Observation of gravitational waves from a binary black hole merger. Phys. Rev. Lett. 116, 6 (2016).

2. https://gcn.gsfc.nasa.gov/gcn3/25333.gcn3.

3. K. Akiyama et al. First M87 event horizon telescope results. I. The shadow of the supermassive black hole. Astrophys. J. 875, (2019).

4. R. Konoplya, L. Rezzolla, A. Zhidenko. General parametrization of axisymmetric black holes in metric theories of gravity. Phys. Rev. D 93, 064015 (2016).

5. J. Abedi, H. Dykaar, N. Afshordi. Echoes from the Abyss: Tentative evidence for Planck-scale structure at black hole horizons. Phys. Rev. D 96, 082004 (2017).

6. A. Mitra, C. Corda, H.J. Mosquera Cuesta. How to distinguish an actual astrophysical magnetized black hole mimicker from a true (theoretical) black hole. http://arxiv.org/abs/1908.06815v1.

7. A. Starobinskii. Amplification of waves during reflection from a rotating "black hole". Zh. Eksp. Teor. Fiz. 64, 48 (1973).

8. W.H. Press, S.A. Teukolsky. Floating orbits, superradiant scattering and the black-hole bomb. Nature 238, 211 (1972).

9. S. Teukolsky. Perturbations of a rotating black hole. I. Fundamental equations for gravitational, electromagnetic, and neutrino-field perturbations. The Astrophysical Journal 185, 635 (1973).

10. A. Starobinsky, S. Churilov. Amplification of electromagnetic and gravitational waves scattered by a rotating black hole. Sov. Phys. - JETP 38, 1 (1974).

11. S. Teukolsky. The Kerr metric. Astrophys. J. 32, 124006 (2015).

12. M. Casals, A. C. Ottewill, N. Warburton. High-order asymptotics for the spin-weighted spheroidal equation at large real frequency. arXiv:1810.00432v1 [gr-qc].

13. B. Mashhoon. Scattering of electromagnetic radiation from a black hole. Phys. Rev. D 7, 2807 (1973). 
14. V. Pelykh, Y. Taistra. A class of general solutions of the Maxwell equations in the Kerr space-time. J. Math. Sci. 229, No. 2, 162 (2018).

15. V. Pelykh, Y. Taistra. Solution with separable variables for null one-way Maxwell field in Kerr space-time. Acta Phys. Polon. Supp. 10, 387 (2017).

16. V. Pelykh, Y. Taistra. Null one-way fields in the Kerr spacetime. Ukr. J. Phys. 62, No. 11, 1007 (2017).

17. V. Pelykh, Y. Taistra. On the null one-way solution to Maxwell equations in the Kerr space-time. Math. Model. Comput. 5, No. 2, 201 (2018).

18. E. Guadagnini. Gravitational deflection of light and helicity asymmetry. Phys. Lett. B 548, Iss. 1-2, 19 (2002).

19. A. Barbieri, E. Guadagnini. Gravitational optical activity. Nucl. Phys. 703, Iss. 1, 391 (2004)

20. V. Frolov, A. Shoom. Scattering of circularly polarized light by a rotating black hole. Phys. Rev. D 86, Iss. 2, 024010 (2012)

21. F. Asenjo, S. Hojman. Do electromagnetic waves always propagate along null geodesics? Class. and Quant. Gravity 34, No. 20, 205011 (2017).

22. S. Chandrasekhar. On algebraically special perturbations of black holes. Proc. R. Soc. London, Ser. A 392, 1 (1984).
23. R. Plyatsko. Manifestations of Gravitational Ultrarelativistic Spin-Orbit Interaction (Naukova Dumka, 1988) (in Ukrainian).

24. R. Plyatsko, M. Fenyk. Highly relativistic circular orbits of spinning particle in the Kerr field. Phys. Rev. D 87, No. 4, 044019 (2013).

Received 02.09.19

B.О. Пелих, Ю.В. Тайстра

ХВИЛЬОВА ОПТИКА У ПРОСТОРІ КЕРРА

З ВРАХУВАННЯМ СПІН-СПІРАЛЬНОЇ ВЗАЄМОДІї

$\mathrm{P}$ е $з$ ю м е

Ми застосовуємо алгебраїчно-спеціальний вихідний в сенсі Чандрасекара розв'язок рівнянь Максвелла у просторі Керра, отримуємо вирази хвильових векторів право- та лівополяризованих хвиль і доводимо, що з умови ізотропності як умови рівності нулеві інваріантів поля не випливає ізотропність хвильових векторів, а також, що інтегральна конгруенція хвильового векторного поля не є геодезійною. Ми встановлюємо зв'язок отриманих результатів із умовою Старобінського та Пресса-Тюкольського існування супервипромінювання в просторі-часі Керра. 